Dear Author,

Please, note that changes made to the HTML content will be added to the article before publication, but are not reflected in this PDF.

Note also that this file should not be used for submitting corrections. 


\title{
An algorithm for beat-to-beat heart rate detection from the BCG based on the continuous spline wavelet transform
}

\author{
Q1 Carlos Alvarado-Serrano ${ }^{\mathrm{a}, *}$, Pablo Samuel Luna-Lozano ${ }^{\mathrm{b}, 1}$, Ramon Pallàs-Areny ${ }^{\mathrm{c}, 2}$ \\ a Bioelectronics Section, Department of Electrical Engineering, Centro de Investigación y de Estudios Avanzados del Instituto Politécnico Nacional México \\ (CINVESTAV), México, D.F., Mexico \\ ${ }^{\mathrm{b}}$ Faculty of Engineering in Electronic Instrumentation, Universidad Veracruzana, Xalapa, Veracruz, Mexico \\ ${ }^{\mathrm{c}}$ Instrumentation Sensors and Interfaces Group, Universitat Politècnica de Catalunya (Barcelona Tech), Barcelona, Spain
}

21 A R T I C L E I N F O

Received in revised form 4 February 2016

Accepted 4 February 2016

Available online xxx

\section{Keywords:}

BCG

Heart rate

Wavelet transform

\begin{abstract}
A B S T R A C T
This work describes an algorithm intended to detect the beat-to-beat heart rate from the ballistocardiogram (BCG) obtained from seated subjects. The algorithm is based on the continuous wavelet transform with splines, which enables the selection of an optimum scale for reducing noise and mechanical interferences. The first step of the algorithm is a learning phase in which the first four heartbeats in the BCG are detected to define initial thresholds, search windows and interval limits. The learned parameters serve to identify the next heartbeat and are readapted after each heartbeat detected to follow the heart rate and signal-amplitude changes. To evaluate the agreement between results from the algorithm and the heart rate obtained from the ECG, a Bland-Altman plot has been used to compare them for seven seated subjects. The mean error obtained was -0.03 beats $/ \mathrm{min}$ and the $95 \%$ confidence interval ( $\pm 2 \mathrm{SD}$ ) was \pm 2.7 beats/min, which is within the accuracy limits recommended by the Association for the Advancement of Medical Instrumentation (AAMI) standard for heart rate meters.
\end{abstract}

(c) 2016 Published by Elsevier Ltd.

\section{Introduction}

The ballistocardiogram (BCG) describes the movement of the center of gravity within the body caused by changes in the mass distribution due to the main circulatory events that occur in the ventricles and in the larger arteries at each heartbeat. These movements can be recorded in terms of displacement, velocity or acceleration [1].

In the 1930s, Starr constructed what is considered the first ballistocardiograph [2], a supine recording system based on the opposition to the movement of the body that attenuated the respiratory content in the record, but despite its high potential value it rapidly fell into disuse in the 1960s. Physicians lost interest

\footnotetext{
* Corresponding author at: CINVESTAV, Departamento de Ingeniería Eléctrica, Sección de Bioelectrónica, Av. Instituto Politécnico Nacional 2508, Col. San Pedro Q2 Zacatenco, México, D.F., 07360, Mexico. Tel.: +52 555747 3800x6216; fax: +52 5557477080 .

E-mail addresses: calvarad@cinvestav.mx (C. Alvarado-Serrano), paluna@uv.mx (P.S. Luna-Lozano), ramon.pallas@upc.edu (R. Pallàs-Areny).

1 Address: Circuito Aguirre Beltrán s/n, Zona Universitaria, Xalapa, Veracruz 91060, Mexico.

2 Address: EETAC, Esteve Terradas 7, Edifici C4, Castelldefels, 08860 Barcelona, Spain.
}

http://dx.doi.org/10.1016/j.bspc.2016.02.002

1746-8094/@ 2016 Published by Elsevier Ltd. in the BCG due to the poor reproducibility of the cardiac cycles even in healthy people and preferred the then recently introduced catheter-based cardiovascular monitoring methods. In the last years, home health monitoring and e-health at large have renewed the interest for research focused on the BCG as a non-obtrusive technique able to assess cardiovascular and respiratory activity [3]. Sensor technology development has enabled the design of low cost recording systems that can be embedded in common objects, such as beds [4], office chairs [5,6], wheelchairs [7] and body weighing scales [8], and enables patients to perform the measurements themselves with minimal medical staff support. These factors minimize stress induced by examinations and the patient involuntary psychophysiological responses [9]. Additionally, computer science enables the development of more powerful signal processing tools able to extract information from complex signals.

The reaction forces that originate the BCG induce body displacement in three directions [10]: head-foot (longitudinal), anterior-posterior (dorsoventral) and left-right (transverse). Fig. 1 shows a longitudinal BCG cardiac cycle recorded in the seat of an office chair [6] and its corresponding electrocardiogram (ECG) simultaneously recorded. This is the most common and easiest component of the BCG that can be obtained in seated and standing position. BCG waves in normal records are named with consecutive letters from $\mathrm{H}$ to $\mathrm{N}$. The $\mathrm{H}$ to $\mathrm{L}$ waves are the main oscillations of 


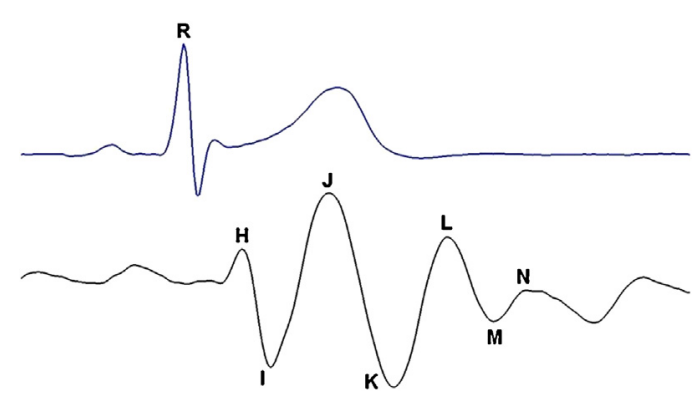

Fig. 1. R-wave (R) of the ECG waveform (top) and BCG waveform of a cardiac cycle measured in the longitudinal direction in the seat of an office chair (bottom). BCG waves are named from the $\mathrm{H}$ (first positive deflection after QRS complex of the ECG) to the $\mathrm{N}$.

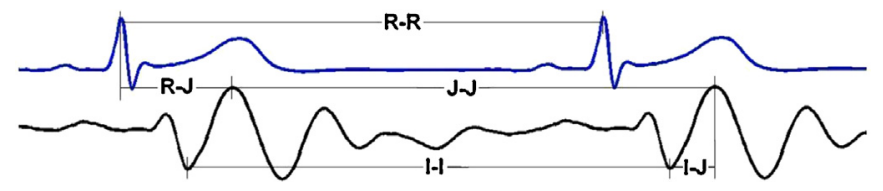

Fig. 2. Time intervals from one cardiac cycle to another in the ECG (top) and the BCG (bottom). R-R (ECG interval between consecutive R-wave peaks), R-J (interval from the ECG R-wave peak to the BCG J-wave peak), J-J (BCG interval between consecutive J-wave peaks), I-I (BCG interval between consecutive I-wave peaks) and I-J (BCG interval from I-wave peak to J-wave peak).

the BCG because of its good reproducibility and larger magnitude, being the $\mathrm{J}$ wave the central and largest positive deflection. It is noted that J-wave peak $\left(J_{\mathrm{p}}\right)$ appears close to the T-wave peak in the ECG. In automated BCG analysis, the detection of $J_{\mathrm{p}}$ is important because once it has been detected, other BCG waves amplitudes and intervals such as J-J or I-J (shown in Fig. 2) can be analyzed with more detail [6].

For heart rate (HR) calculations, the I, J and K waves are the most common waves used, and are related to the ventricular ejection and aortic flow [1]. The I-J amplitude reflects the maximal force delivered by the heart during the ventricular systole in order to propel blood flow [11]. Recently, J-wave amplitude has been proposed to monitor the ejection force in patients with diagnosed heart failure during cardiac resynchronization therapy optimization [12]. The time interval between the R-wave peak of the simultaneously measured ECG and the $J_{\mathrm{p}}$, named R-J interval, has been proposed to estimate the systolic blood pressure [13] and cardiac contractility [14].

Beat-to-beat HR detection in the BCG without using any other signal as a reference is not a trivial task because its waveform strongly changes from subject-to-subject and even from beat-tobeat in the same record of a given subject. These changes can be attributed, at least in part, to internal and external mechanical interferences such as breathing, muscular temblors and vibrations. Several algorithms have been proposed to detect individual heartbeats from the BCG, some based on the search of largest deflection [15] which was very sensitive to motion artifacts. Some others are based on the discrete wavelet transform (DWT) [7], for which is required additional signal processing for noise filtering; and the continuous wavelet transform (CWT) [16], that required two ranges of scales to obtain low and high HR. Other algorithms that involve a high computational load due to its complexity are based on unsupervised learning techniques [4], continuous local interval estimation with three estimators combined [17] and adaptive heartbeat shape modeling [18].

Regarding the wavelet transform, it is a suitable tool that has been used to determine peaks and limits of ECG waves because of its ability to detect transients and of its robustness in front of noise and artifacts [19]. Wavelet transforms at different scales describe the time characteristics of a signal in different frequency bands, but the analysis is restricted to scales that are powers of two [20]. The use of B-splines as base functions permits the evaluation of the CWT in any integer scale [21], which enables to use a wider range of scales and to reduce noise and artifacts more efficiently. This feature can allow the direct application of the algorithm over the raw BCG signal without any pre-processing stage, because frequency filtering is performed when the CWT is computed. In this work, an algorithm to detect $J_{\mathrm{p}}$ in the BCG based on the CWT with splines is presented, which has been evaluated in seven BCG records obtained in seated subjects.

\section{Materials and methods}

\subsection{BCG and ECG acquisition}

The longitudinal BCG was recorded in 7 seated subjects ( 5 males, 2 females; age $33 \pm 6$ years) with different physical constitution (weight and height ranges from 67 to $87 \mathrm{~kg}$ and from 1.65 to $1.75 \mathrm{~m}$ respectively). The ballistic force was measured with a piezoelectric sensor fixed with adhesive tape to the bottom side of the seat of a common office chair [6]. Fig. 3 shows the chair, the location of the sensor, and the block diagram of the force measurement system. The mechanical stress of the seat yields electrical charge variations at the sensor output and a charge amplifier converted these to voltage signals.

To reduce noise and mechanical interferences, BCG was band limited. The charge amplifier has a first order high pass response, which was settled to $f_{L}=0.5 \mathrm{~Hz}$. The bias current of this amplifier produces an offset voltage at the output of the amplifier even though the high pass response. This was canceled by a passive first order high pass filter (HPF) with the same cutoff frequency, therefore, the overall high pass response of the circuit was of second order $(n=2)$. The output of the passive HPF was band limited with a second order low pass filter (LPF) to $f_{H}=20 \mathrm{~Hz}$ with an active filter. Finally, the BCG was digitized at a sampling rate of $1 \mathrm{kS} / \mathrm{s}$ with 12-bit resolution.

The ECG (lead I) was simultaneously recorded as a reference signal but otherwise it was not used to process the BCG. It was amplified by 1340 to fit the dynamic range of the ADC and band limited with active filters between $0.16 \mathrm{~Hz}$ (with a second order $\mathrm{HPF}$ ) and $100 \mathrm{~Hz}$ (with a third order LPF). BCG and ECG acquisition systems used a ground isolated power supply of $\pm 6 \mathrm{~V}$.

\subsection{Wavelet transform}

The wavelet transform decomposes a signal in components that appear at different scales or resolutions [20]. Thus, the CWT of a time-continuous signal $x(t)$ is defined as

$\operatorname{CWT}\{x(t) ; a, b\}=\frac{1}{\sqrt{a}} \int_{-\infty}^{\infty} x(t) \psi^{*}\left(\frac{t-b}{a}\right) d t$

where $\psi^{*}(t)$ is the complex conjugate of the analyzing wavelet function $\psi(t)$ and $a$ and $b$ are the scale and translation parameters respectively. The function $\psi(t)$ compresses or dilates depending on $a$, which enables the CWT to extract the low and high-frequency components of $x(t)$. To implement the CWT, $a$ and $b$ are usually discretized. If $a$ is discretized over a sequence $2^{j}(j=1,2, \ldots)$, the analysis is restricted to scales that are powers of two, and the result is the dyadic wavelet transform that can be computed with Mallat's algorithm [20].

In this work, B-splines have been used which enable the evaluation of the CWT in any integer scale [21]. In this approach, the input signal $x(t)$ and the analyzing wavelet $\psi(t)$ are both represented by 

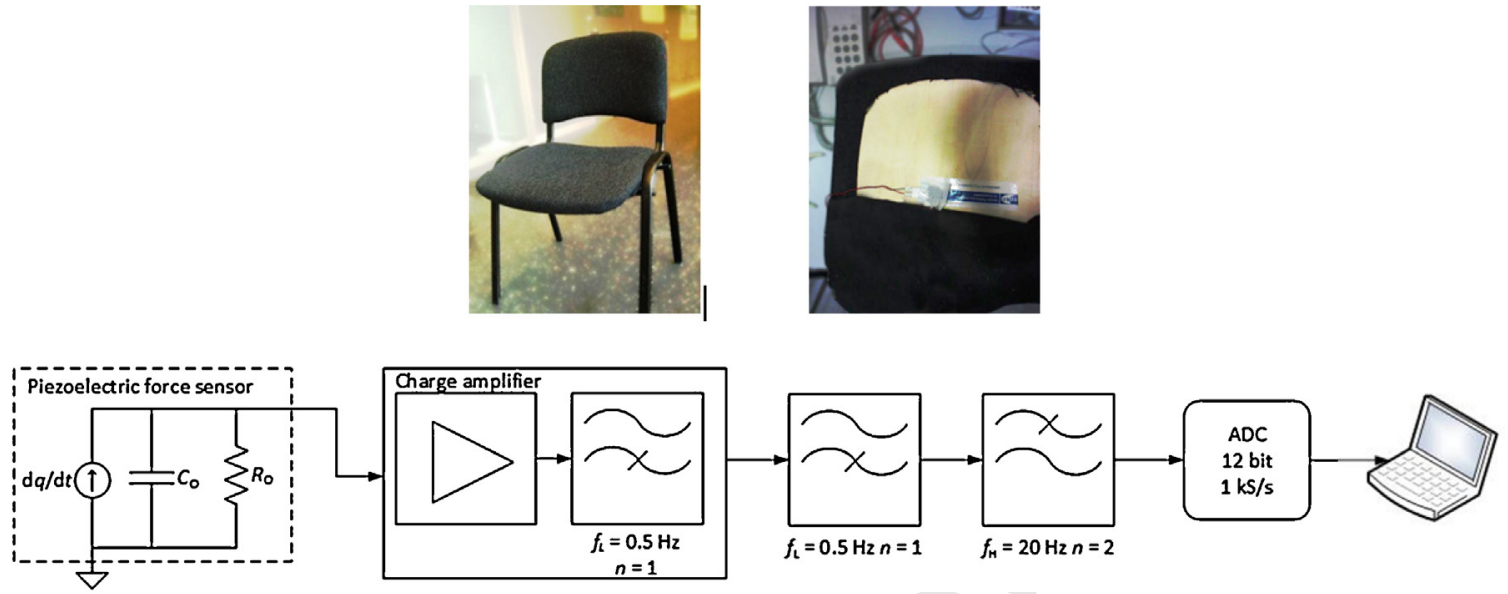

Fig. 3. BCG acquisition system. Chair used and location of piezoelectric force sensor (top) and block diagram of the force measurement system (bottom).

polynomial splines of degree $n_{1}$ and $n_{2}$ respectively, and therefore the resulting CWT will be a polynomial spline function.

The splines are functions constructed from polynomial segments of degree $n$ of unit length that are smoothly connected together at joining points called knots, in a way such that guarantees the continuity of the function and its derivatives up to order $(n-1)$.

The B-splines are the basic building blocks for splines and are bell-shaped functions of compact support that are constructed from the repeated convolution of a centered unit rectangular pulse (Bspline of degree zero) $\beta^{0}(t)$

$\beta^{n}(t)=\beta^{0} \times \beta^{n-1}(t)$

Input signal $x(t)$ is characterized in terms of its B-spline expansion of degree $n_{1}$ and the sequence of B-spline coefficients $c(k)$

$x(t)=\sum_{k \in Z} c(k) \beta^{n_{1}}(t-k)$

Then, the wavelet $\psi(t)$ is a spline of degree $n_{2}$ with its B-spline expansion

$\psi(t)=\sum_{k \in Z} p(k) \beta^{n_{2}}(t-k)$

B-splines satisfy a two-scale equation for any integer $m$, where $m$ is not restricted to a power of two, thus the wavelet expanded by a factor $m$ can be expressed as

$\psi(t / m)=\sum_{k \in Z}\left([p]_{\uparrow m} \times u_{m}^{n_{2}}\right)(k) \beta^{n_{2}}(t-k)$

where the sequence $u_{m i}^{n_{2}}(k)$, when $n_{2}$ and $m$ are not both even, is given by $z$ transform,

$u_{m}^{n_{2}}(z)=\frac{z^{k_{0}}}{m^{n_{2}}}\left(\sum_{k=0}^{m-1} z^{-k}\right)^{n_{2}+1}$

with $k_{0}=\left(n_{2}+1\right)(m-1) / 2$.

The resulting CWT at scale $m$ evaluated at integer time samples is a polynomial spline function given by

$\mathrm{CWT}\{x(t), m, k\}=\left([p] \uparrow_{m} \times u_{m}^{n_{2}} \times b^{n_{1}+n_{2}+1} \times c\right)(k)$

where the notation $\left([p] \uparrow_{m} \times u_{m}^{n_{2}}\right)(k)$ represents the $k$ th term of the convolution of the sequences $p$ upsampled by a factor $m$ and of sequence $u_{m}^{n_{2}}$, the filter $u_{m}^{n_{2}}$ is a cascade of $\left(n_{2}+1\right)$ moving sum filters of order $(m-1)$ with an offset $k_{0}$ that ensures its symmetry, $b^{n_{1}+n_{2}+1}$ is the B-spline representation of a spline of order $n_{1}+n_{2}+1$, and $c(k)$ 's are the B-splines coefficients.

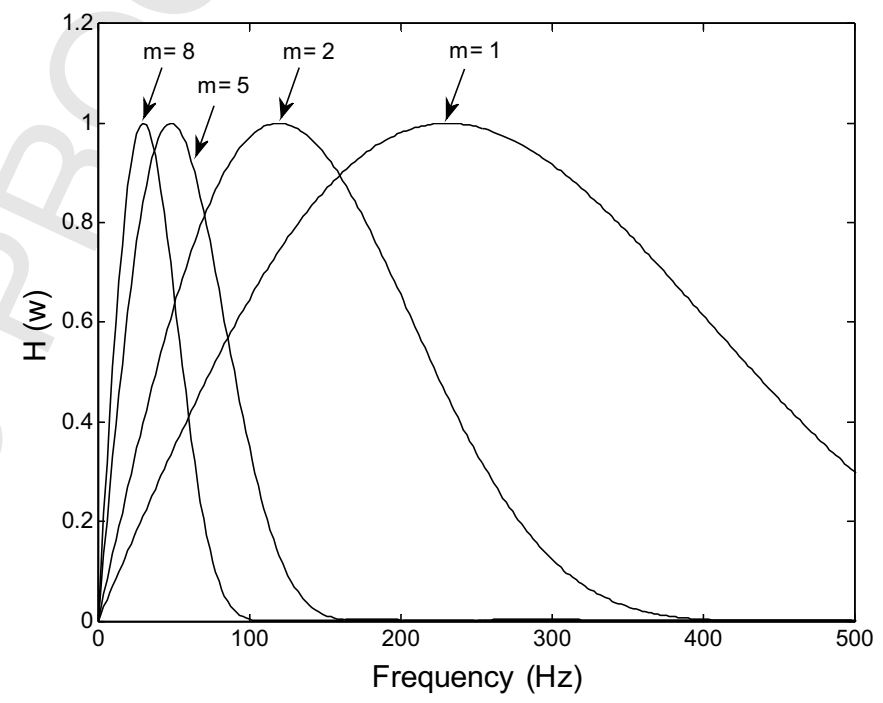

Fig. 4. Amplitude-frequency responses of equivalent filters at scales $m=1,2,5$ and 8 for $1000 \mathrm{~Hz}$ sampling rate $(\mathrm{H}(\mathrm{w})=$ FFT magnitude, $m=$ scale $)$.

The program developed by Alvarado et al. [22] (written in Matlab $^{\circledR}$, The MathWorks Inc.), calculates the CWT of the discrete signal $x(t)$ at the integer scales $m$ with expansion coefficients spline $p$, and its implementation is based in the fast algorithm proposed by Unser et al. [21]. It can be seen from (6) that filters are iterated discrete convolutions of moving sums, and can be computed without any multiplication, which results in a very efficient algorithm.

The selected wavelet function $\psi(t)$ is the first derivative of a 4th-order cubic B-spline expanded by two, which is similar to the first derivative of a Gaussian function and it yields good time and frequency resolution [21]. The Fourier transform of the wavelet at four scales at a sampling frequency of $1 \mathrm{kHz}$ is shown in Fig. 4.

From the equivalent filters responses in Fig. 4, scale 5, which corresponds to a $-3 \mathrm{~dB}$ bandwidth of $23-78 \mathrm{~Hz}$, was selected to reduce the influence of noise, mechanical interferences and detection errors in all BCG records.

\subsection{J-wave peak detection algorithm}

According to the form of the wavelet function selected, the J wave of the BCG corresponds to a pair of local maxima $W_{\mathrm{p}}$ and minima $W_{\mathrm{n}}$ of the modulus $P_{\mathrm{mm}}$ of the CWT by using the scale 5 $\left(\mathrm{CWT}_{5}\right)$, where the rising slope yields a minimum and the falling 


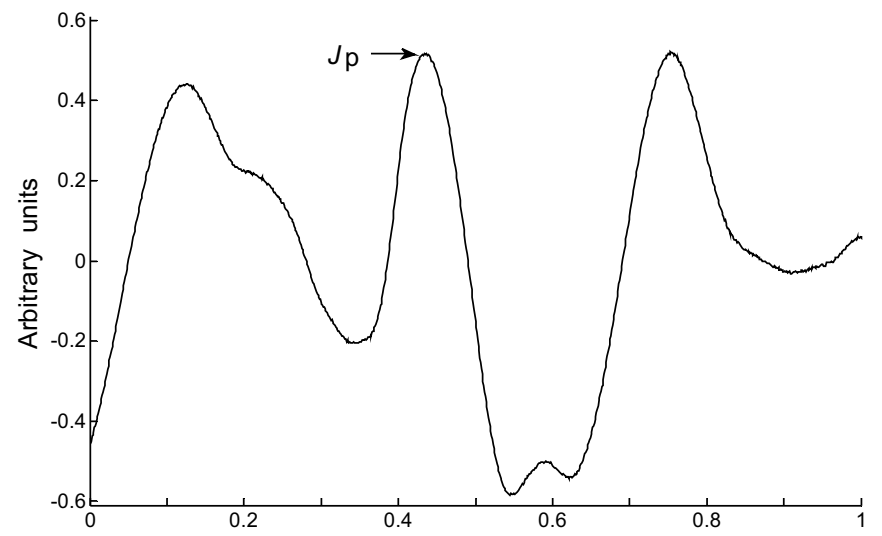

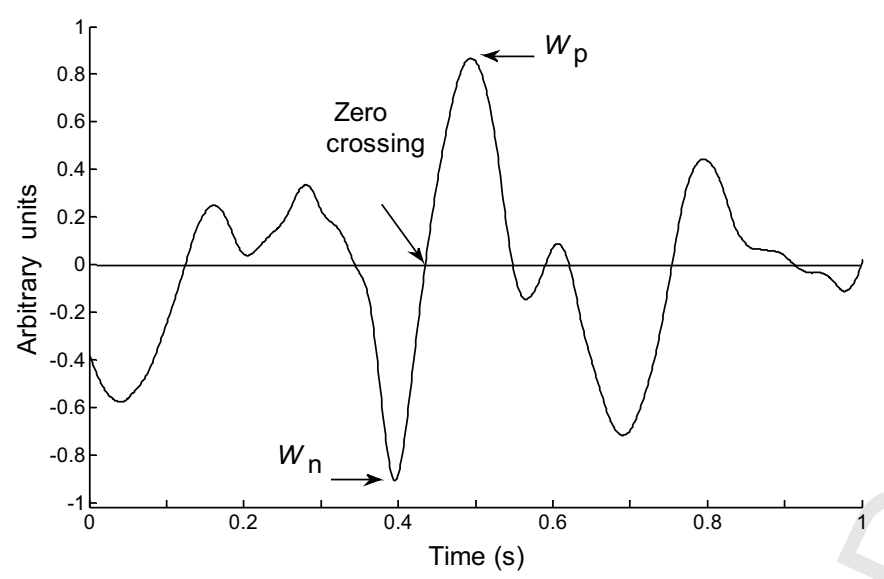

Fig. 5. J-wave peak $\left(J_{\mathrm{p}}\right)$ of the BCG (top) and positive maxima $\left(W_{\mathrm{p}}\right)$ and negative minima $\left(W_{n}\right)$ of the CWT at scale 5 of the BCG (bottom). slope yields a maximum [19]. $J_{\mathrm{p}}$ corresponds to the zero crossing of $P_{\mathrm{mm}}$ (Fig. 5).

The algorithm for $J_{\mathrm{p}}$ detection is directly applied to the CWT of the longitudinal BCG band limited and comprises two stages: learning and decision.

\subsubsection{Learning stage}

The learning stage includes the definition of a first set of detection thresholds to detect the first four beats considered as $J_{\mathrm{p}}$, a refractory period of $300 \mathrm{~ms}$ to avoid false detections once $J_{\mathrm{p}}$ has been located [23], and the calculus of a J-J interval average obtained of the first three J-J intervals to define an initial search window. ative peak $\left(W_{\mathrm{n}}\right)$ are searched within the first $2 \mathrm{~s}$ of the record in order to define a positive threshold $\left(T_{\mathrm{p} 1}=K_{\mathrm{p}} W_{\mathrm{p}}\right)$ and a negative threshold $\left(T_{\mathrm{n} 1}=K_{\mathrm{n}} W_{\mathrm{n}}\right)$. Constants $K_{\mathrm{p}}$ and $K_{\mathrm{n}}$ are experimental values that in the algorithm performs best for $K_{\mathrm{p}}=0.5$ and $K_{\mathrm{n}}=0.4$. Thereafter, in the first $2 \mathrm{~s}$ a positive peak larger than $T_{\mathrm{p} 1}\left(W_{\mathrm{p}}(1: 4)\right)$ and its nearest negative peak backwards lower than $T_{\mathrm{n} 1}\left(W_{\mathrm{n}}(1: 4)\right)$ are searched. A zero crossing between those points would be the first beat considered $J_{\mathrm{p}}(1: 4)$. With this method, the following three beats considered as $J_{\mathrm{p}}$, are detected to calculate the first three values of J-J interval, $\mathrm{JJ}(1: 3)$. Then, an average value defined as $J J_{\mathrm{av}}(1: 3)=K_{\mathrm{sw}} \times \operatorname{mean}[J J(1: 3)]$ is used as the initial value to define a search window for the next $J_{\mathrm{p}}$ detection, where constant $K_{\mathrm{sw}}$ is an experimental value that in the algorithm has its best performance for $K_{\mathrm{sw}}=0.8$. Also, the first three HR values are calculated.
In this stage, the highest positive peak $\left(W_{\mathrm{p}}\right)$ and the lowest neg-

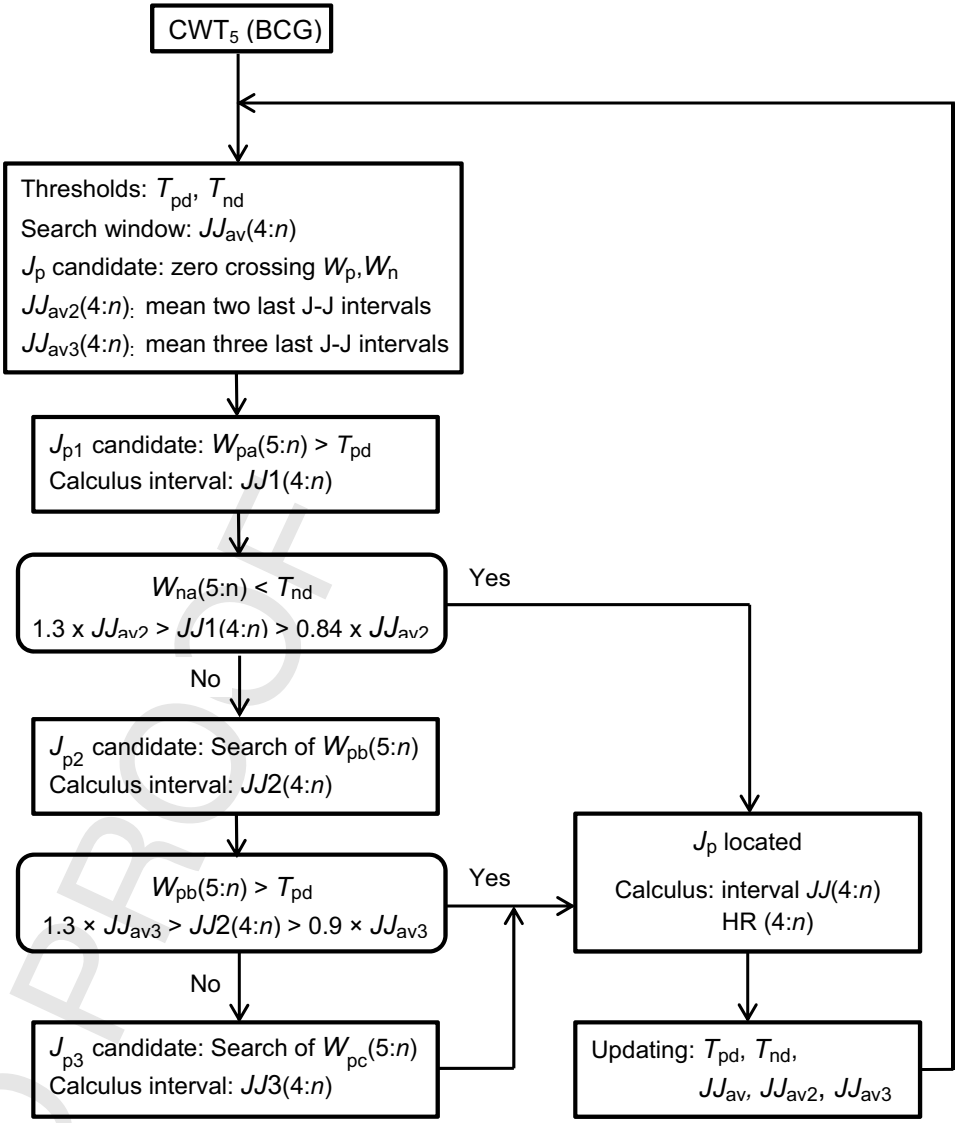

Fig. 6. Flowchart of the decision stage of the $J_{\mathrm{p}}$ detection algorithm.

\subsubsection{Decision stage}

The decision stage is a process of three steps to recognize $J_{\mathrm{p}}$. It includes the definition of a second set of adaptive detection thresholds and an adaptive search window, besides of the calculus of two $\mathrm{J}-\mathrm{J}$ intervals average that are the mean of the two and three last $\mathrm{J}-\mathrm{J}$ intervals respectively. Flowchart of the decision stage is shown in Fig. 6.

In the first step, positive and negative thresholds are defined as $T_{\mathrm{pd}}=K_{\mathrm{pd}} T_{\mathrm{p} 1}(n)$ and $T_{\mathrm{nd}}=K_{\mathrm{nd}} T_{\mathrm{n} 1}(n)$ respectively, where constants $K_{\mathrm{pd}}$ and $K_{\mathrm{nd}}$ are experimental values that in the algorithm performs best for $K_{\mathrm{pd}}=0.7$ and $K_{\mathrm{nd}}=0.5$. A positive peak larger than $T_{\mathrm{pd}} ;\left(W_{\mathrm{pa}}(5: n)\right)$ and its nearest negative peak backwards $\left(W_{\mathrm{na}}(5: n)\right)$ are searched in the defined window. A zero crossing between those points would be the first $J_{\mathrm{p}}$ candidate $J_{\mathrm{p} 1}(5: n)$ and its corresponding interval $J J 1(4: n)$ is calculated. To determine whether $J_{\mathrm{p}}$ has occurred or not, Eqs. (8) and (9) are applied.

$W_{\text {na }}(5: n)<T_{\text {nd }}$

$1.3 \times J J_{\mathrm{av} 2}>J J 1(4: n)>0.84 \times J J_{\mathrm{av} 2}$

where $J J_{\mathrm{av} 2}$ is the mean of the two most recent intervals $[J J(1: n): J J(2: n)]$.

If these two conditions are not met, in the second step another $J_{\mathrm{p}}$ candidate $J_{\mathrm{p} 2}(5: n)$, the zero crossing between a pair of local maxima, $W_{\mathrm{pb}}(5: n)$ and minima $W_{\mathrm{nb}}(5: n)$, is searched from $J_{\mathrm{p} 1}$ in a new window, and a new interval $J J 2(4: n)$ is calculated. The decision about if a $J_{\mathrm{p}}$ was found, is taken with Eqs. (10) and (11).

$W_{\mathrm{pb}}(5: n)>T_{\mathrm{pd}}$

$1.3 \times J J_{\mathrm{av} 3}>J J 2(4: n)>0.9 \times J J_{\mathrm{av} 3}$

where $J J_{\mathrm{av} 3}$ is the mean of the three most recent J-J intervals $[J J(1: n): J J(3: n)]$. 
Table 1

Results for the proposed $J_{\mathrm{p}}$ detection algorithm applied to seven records of seated subjects and HR ranges (HR in beats/min).

\begin{tabular}{lllc}
\hline Subject & Beats & HR $(\min )$ & HR (max) \\
\hline 1 & 119 & 66 & 78 \\
2 & 58 & 55 & 64 \\
3 & 123 & 70 & 80 \\
4 & 131 & 63 & 103 \\
5 & 109 & 59 & 77 \\
6 & 105 & 54 & 77 \\
7 & 130 & 75 & 91 \\
Total & 775 & 54 & 103 \\
\hline
\end{tabular}

If these second pair of conditions are not fulfilled, in the third step the last $J_{\mathrm{p}}$ candidate $J_{\mathrm{p} 3}(5: n)$, the zero crossing between a pair of local maxima, $W_{\mathrm{pc}}(5: n)$ and minima $W_{\mathrm{nc}}(5: n)$, is searched from $J_{\mathrm{p} 2}$ in a new window, and a last interval $J J 3(4: n)$ is calculated. In this step $J_{\mathrm{p} 3}(5: n)$ is considered to be $J_{\mathrm{p}}$, and $J J 3(4: n)$ is a valid interval.

In any of the steps once $J_{\mathrm{p}}$ is validated, $\mathrm{HR}$ values are calculated and adaptive detection thresholds and an adaptive search window are defined. The next thresholds at the $n$th beat are calculated as

$T_{\mathrm{pd}}(n+1)=0.4 T_{\mathrm{pd}}(n)+0.2 W_{\mathrm{p}}(n)$

$T_{\text {nd }}(n+1)=0.4 T_{\text {nd }}(n)+0.2 W_{\mathrm{n}}(n)$

For the search of $J_{\mathrm{p}}$ at the $n$th beat, the next search windows $J J a v(n+1)$ are calculated from the last J-J interval $J J(n)$, as described by Laguna et al. [24] for QT interval measurement, but the low and high J-J limits are modified as follows in order to adapt to heart rate changes,

$$
\text { If } \quad \begin{array}{ll}
1.2 J J_{\mathrm{av}}(n)>J J(n)>0.8 J J_{\mathrm{av}}(n) \\
& J J J a v_{\mathrm{av}}(n+1)=0.8 J J_{\mathrm{av}}(n)+0.2 J J(n)
\end{array}
$$

else

$J J a v(n+1)=J J a v(n)$

\section{Results and discussion}

The algorithm for $J_{\mathrm{p}}$ detection based on the CWT with splines above described has been tested in seven longitudinal BCG records (six lasting for $100 \mathrm{~s}$ and one lasting for $60 \mathrm{~s}$ ) on seated subjects. R-R intervals from the simultaneous recorded ECG were obtained with an algorithm for QRS detection based on the CWT with splines using the scale 2 , which has an accuracy of 99.5\% [22]. To quantitatively evaluate the algorithm, the agreement between R-R intervals from ECG and J-J intervals from BCG and their corresponding heart rates were assessed by using the statistical method proposed by Bland and Altman [25].

Table 1 shows the beats detected by the algorithm (number of $J_{p}$ in each record) and the HR range calculated for each subject. In total, 775 beats were detected in a range of HR from 54 to 103 beats/min.

Fig. 7 shows the behavior of the $J_{\mathrm{p}}$ detection algorithm in the record of the subject \#4. Fig. 7(a) shows ECG heart rate changes with QRS and $J_{\mathrm{p}}$ detections. The algorithm follows beat-to-beat changes of HR from 63 to 103 beats/min. In Fig. 7(b), one segment of the BCG record with $J_{\mathrm{p}}$ and QRS detections is shown to illustrate the robustness of the algorithm when the amplitude of the J wave changes; $J_{\mathrm{p}}$ and QRS detections are identified by a cross and square respectively. Fig. 7(c) shows Bland-Altman plot in which the mean error of the intervals obtained from each signal, was $0.05 \mathrm{~ms}$ and the $95 \%$ confidence interval ( $\pm 2 \mathrm{SD}$ ) was $\pm 24.2 \mathrm{~ms}$.

Bland-Altman method was applied to the seven records of seated subjects with a total of 775 intervals measured for each signal, with a mean bias of $0.01 \mathrm{~ms}$ and a $95 \%$ confidence inter$\mathrm{val}( \pm 2 \mathrm{SD}$ ) of $\pm 28.7 \mathrm{~ms}$. There is no systematic bias in the intervals (a)

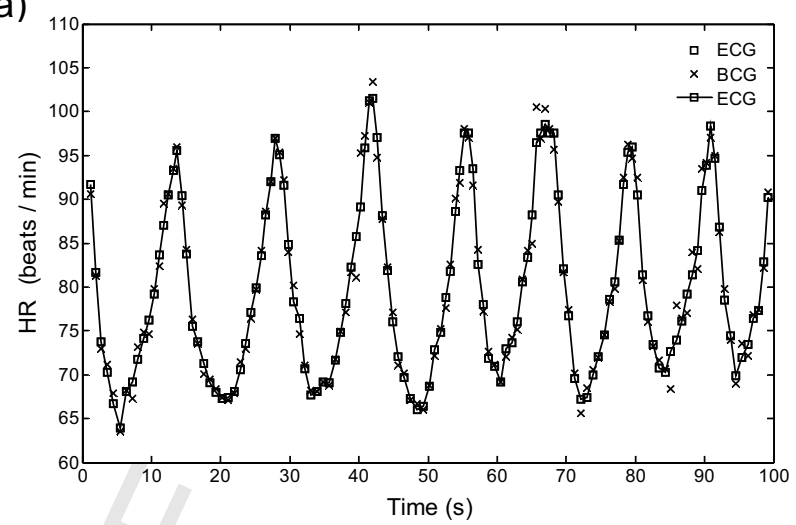

(b)

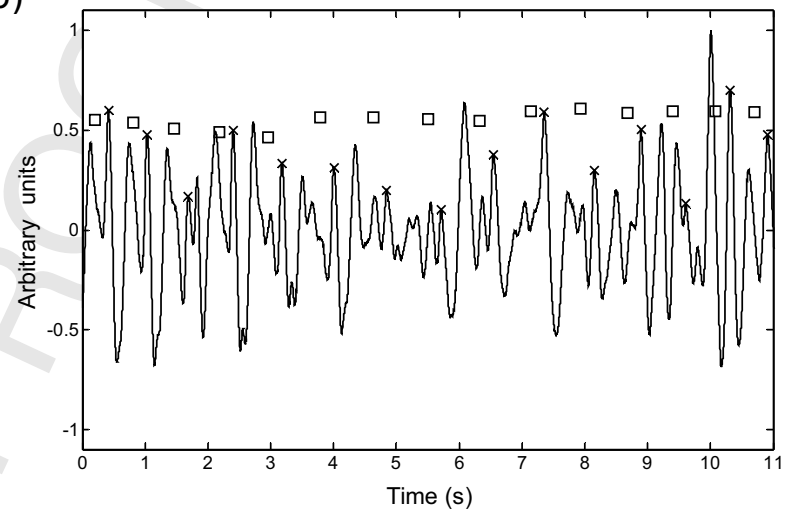

(c)

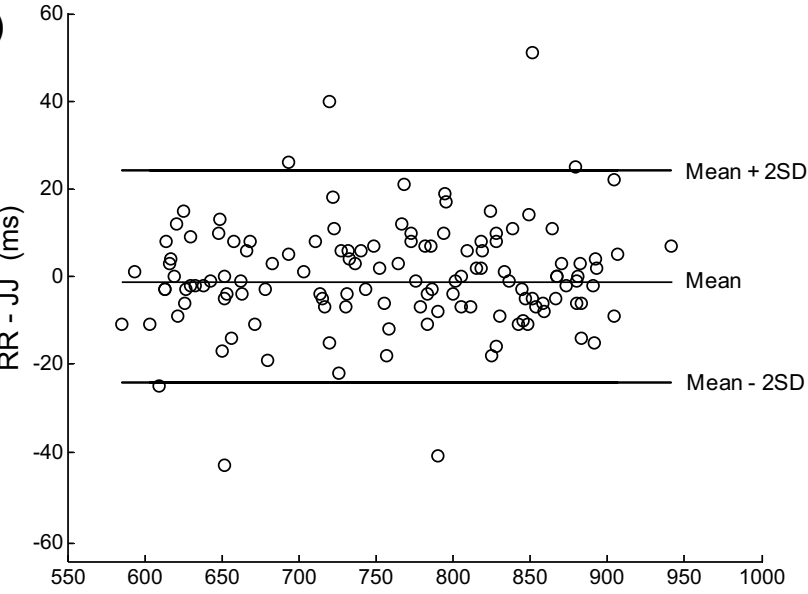

Average of RR interval from ECG and JJ interval from BCG (ms)

Fig. 7. Behavior of the $J_{\mathrm{p}}$ detection algorithm in the subject \#4. (a) ECG heart rate changes with QRS and $J_{\mathrm{p}}$ detections, (b) BCG with $J_{\mathrm{p}}$ and QRS detections, and (c) Bland-Altman plot of average of RR and JJ intervals measured from ECG and BCG signals respectively against its difference.

obtained with the proposed algorithm and the standard deviations are comparable to those obtained in other BCG analysis $[4,8,17,18]$, hence validating the algorithm proposed.

The mean error of the heart rates obtained from each signal, was 0.03 beats $/ \mathrm{min}$ and the $95 \%$ confidence interval $( \pm 2 \mathrm{SD})$ was \pm 2.7 beats/min as shown in Bland-Altman plot (Fig. 8), which is within the accuracy limits of \pm 5 beats/min recommended by the Association for the Advancement of Medical Instrumentation (AAMI) standard for heart rate meters [26].

Regarding the complexity of the algorithm, in the preprocessing stage the use of B splines for the fast implementation of the CWT achieves the lowest complexity per computed coefficient [27]; in 


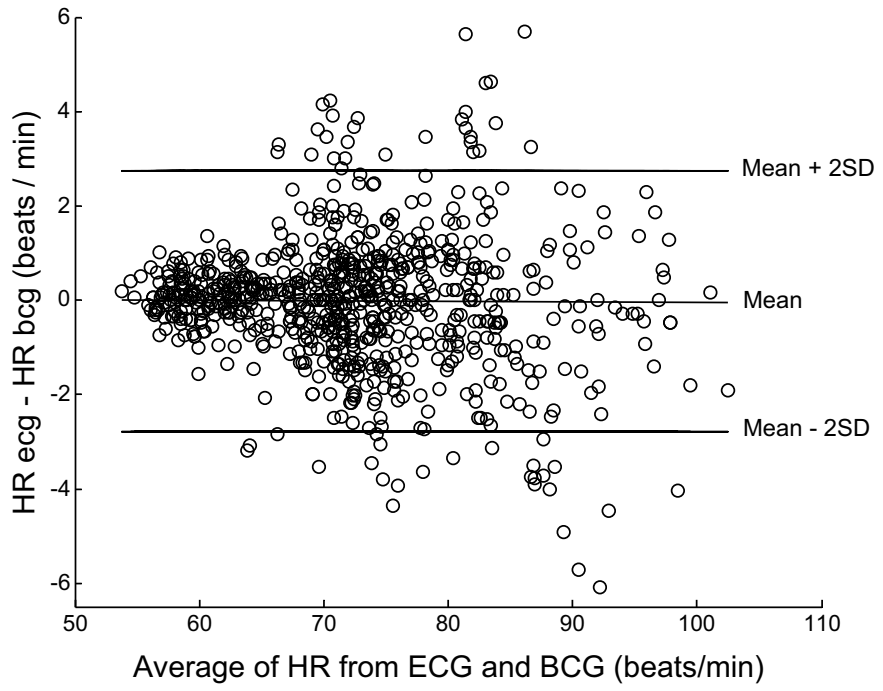

Fig. 8. Bland-Altman plot of average of heart rates measured from ECG and BCG signals against its difference in seven seated subjects.

the learning and decision stages the algorithm uses simple signal processing techniques and heuristic rules of a lower complexity than other algorithms $[4,17,18]$.

$J_{\mathrm{p}}$ detections outside of the limits of agreement $( \pm 28.7 \mathrm{~ms})$ are randomly distributed, they can be caused by internal and external mechanical interferences and by changes in the R-J interval. Comparisons of BCG and ECG interval estimates must consider that the mechanical and electrical activities involved are not identical $[17,28]$ because the R-J interval undergoes physiological-induced changes. Previous works have shown that changes in the R-J interval reflect sympathetic and parasympathetic influence on the contractility of the myocardium and the effect of cardiac filling [29]. Further, these changes are correlated to changes in the preejection period caused by Valsalva maneuver and tilt tests $[14,30]$ and the systolic blood pressure [13]. Etemadi et al. [14] reported that the mean R-J interval was $245 \mathrm{~ms}$ with a standard deviation of $20 \mathrm{~ms}$.

\section{Conclusion}

HR detection from the BCG is a difficult task due to waveform changes from subject to subject and even from beat to beat in the same record of a given subject. To overcome this problem, a detection algorithm of $J_{\mathrm{p}}$ based on the CWT with splines has been proposed that can reduce the effects of noise, baseline drifts and mechanical interferences very common in the BCG, by an appropriate scale selection. To improve the detection and automatically adapt it to HR and amplitude changes, the algorithm uses techniques drawn from previous work on ECG processing that have been conveniently modified in the learning and decision stages, adaptive thresholds and search windows and refractory blanking periods.

The performance of the beat-to-beat heart rate estimations from the BCG was evaluated by using a simultaneous ECG as a reference in seven seated subjects. The accuracy is comparable to results from other BCG analysis algorithms, but the proposed algorithm requires lower computational load, and is within the accuracy limits recommended by AAMI standard for heart rate meters. In order to improve the accuracy, future research could compare J-J intervals of the BCG to T-T intervals of the ECG because $J_{\mathrm{p}}$ almost agrees with T wave peak of the ECG. The beat to beat interval information provided by the algorithm can be used, for example, for applications such as R-J and J-J intervals variability analysis.

\section{Acknowledgments}

This work was supported in part by the Spanish Ministry of Q4 363 Science and Innovation under Grant TEC2009-13022 and by the European Fund for Regional Development. C. Alvarado-Serrano thanks G. Vega-Martinez for his help with the presentation of the statistical method. Authors thank the volunteers for their patience and cooperation.

\section{References}

[1] A. Noordergraaf, C.E. Heynekamp, Genesis of displacement of the human longitudinal ballistocardiogram from the changing blood distribution, Am. J. Cardiol. 2 (1958) 748-756.

[2] N.T. Smith, Ballistocardiography, in: A.M. Weissler (Ed.), Noninvasive Cardiology, Grune \& Stratton, New York, 1974, pp. 39-148.

[3] K. Yamakoshi, In the spotlight: bioinstrumentation, IEEE Rev. Biomed. Eng. 2 (2009) 2-5.

[4] C. Brüser, K. Stadlthanner, S. de Waele, S. Leonhardt, Adaptive beat-to-beat heart rate estimation in ballistocardiograms, IEEE Trans. Inf. Technol. Biomed. 15 (2011) 778-786.

[5] S. Junnila, A. Akhbardeh, A. Värri, An electromechanical film sensor based wireless ballistocardiographic chair: implementation and performance, J. Signal Process. Syst. 57 (2009) 305-320.

[6] P.S. Luna-Lozano, C. Alvarado-Serrano, Time and amplitude relationships of the ballistocardiogram in vertical and horizontal direction, in: Proceedings of the 9th Int. Conf. on Elec. Eng. Comput. Sci. and Autom. Control (CCE), Mexico City, Mexico, 2012, pp. 189-194.

[7] O. Postolache, P.S. Girao, J. Mendes, E.C. Pinheiro, G. Postolache, Physiological parameters measurement based on wheelchair embedded sensors and advanced signal processing, IEEE Trans. Instrum. Meas. 59 (2010) 2564-2574

[8] R. González-Landaeta, O. Casas, R. Pallàs-Areny, Heart rate detection from an electronic weighing scale, Physiol. Meas. 29 (2008) 979-988.

[9] G.G. Berntson, J.T. Cacioppo, K.S. Quigley, V.T. Fabro, Autonomic space and psychophysiological response, Psychophysiology 31 (1994) 44-61.

[10] W.R. Scarborough, S.A. Talbot, Proposals for ballistocardiographic nomenclature and conventions: revised and extended: report of committee on ballistocardiographic terminology, Circulation 14 (1956) 435-450.

[11] I. Starr, O. Horwitz, R.L. Mayock, E.B. Krumbhaar, Standardization of the ballis tocardiogram by simulation of the heart's function at necropsy; with a clinical method for the estimation of cardiac strength and normal standards for it, Circulation 1 (1950) 1073-1096.

[12] L. Giovangrandi, O.T. Inan, R.M. Wiard, M. Etemadi, G.T.A. Kovacs, Ballistocardiography - a method worth revisiting, in: Proceedings of the 33rd Annu. Int. Conf. of the IEEE EMBS, Boston, Massachusetts, USA, 2011, pp. 4279-4282.

[13] J.H. Shin, K.M. Lee, K.S. Park, Non-constrained monitoring of systolic blood pressure on a weighing scale, Physiol. Meas. 30 (2009) 679-693.

[14] M. Etemadi, O.T. Inan, L. Giovangrandi, G.T.A. Kovacs, Rapid assessment of cardiac contractility on a home bathroom scale, IEEE Trans. Inf. Technol. Biomed. 15 (2011) 864-869.

[15] B.H. Choi, G.S. Chung, J.-S. Lee, D.-U. Jeong, K.S. Park, Slow-wave sleep estimation on a load-cell-installed bed: a non-constrained method, Physiol. Meas. 30 (2009) 1163-1170.

[16] S. Gilaberte, J. Gómez-Clapers, R. Casanella, R. Pallàs-Areny, Heart and respiratory rate detection on a bathroom scale based on the ballistocardiogram and the continuous wavelet transform, in: Proceedings of the 32nd Annu. Int. Conf. of the IEEE EMBS, Buenos Aires, Argentina, 2010, pp. 2557-2560.

[17] C. Brüser, S. Winter, S. Leonhardt, Robust inter-beat interval estimation in cardiac vibration signals, Physiol. Meas. 34 (2013) 123-138.

[18] J. Paalasmaa, H. Toivonen, M. Partinen, Adaptive heartbeat modelling for beatto-beat heart rate measurement in ballistocardiograms, IEEE J. Biomed. Health Inform. doi: 10.1109/JBHI.2014.2314144.

[19] C. Li, C. Zheng, C. Tai, Detection of ECG characteristic points using wavelet transforms, IEEE Trans. Biomed. Eng. 42 (1995) 21-28.

[20] S.G. Mallat, A theory for multiresolution signal decomposition: the wavele representation, IEEE Trans. Pattern Anal. Mach. Intell. 11 (1989) 674-693.

[21] M. Unser, A. Aldroubi, S.J. Schiff, Fast implementation of the continuous wavelet transform with integer scales, IEEE Trans. Signal Process. 42 (1994) 3519-3523.

[22] C. Alvarado, J. Arregui, J. Ramos, R. Pallàs-Areny, Automatic detection of ECC ventricular activity waves using continuous spline wavelet transform, in Proceedings of the 2nd. Int. Conf. on Elec. and Electron. Eng. (ICEEE), Mexico City, Mexico, 2005, pp. 189-192.

[23] J. Pan, W.J. Tompkins, A real-time QRS detection algorithm, IEEE Trans. Biomed Eng. 32 (1985) 230-236.

[24] P. Laguna, N.V. Thakor, P. Caminal, R. Jané, H.-R. Yoon, A. Bayés de Luna, V. Marti, J. Guindo, New algorithm for QT interval analysis in 24-hour Holter ECG: performance and applications, Med. Biol. Eng. Comput. 28 (1990) 67-73.

[25] J.M. Bland, D.G. Altman, Statistical methods for assessing agreement between two methods of clinical measurement, The Lancet 327 (1986) 307-310.
364
365
366
367
368

371

371

373
374

374
375

376
377

377
378

37

380
381

381
382

383

384
385

385

386

38

389

390

392

393

394
395

39

396
397

39

40

40

403

404
405

405
406

407

408
409

409

41

41

41

414
415

416

417

418
419

420

421

422
423

423

424
425

426

427 
[26] ANSI/AAMI EC13:2002, Cardiac monitors, heart rate meters, and alarms, ANSI Standard, 2002.

[27] M. Unser, Splines: a perfect fit for signal and image processing, IEEE Signal Process. Mag. 16 (1999) 22-38.

[28] D. Friedrich, X.L. Aubert, H. Führ, A. Brauers, Heart rate estimation on a beatto-beat basis via ballistocardiography - a hybrid approach, in: Proceedings of the 32nd Annu. Int. Conf. of the IEEE EMBS, Buenos Aires, Argentina, 2010, pp. 4048-4051.
[29] A. Lindqvist, K. Pihlajamäki, J. Jalonen, V. Laaksonen, J. Alihanka, Static-chargesensitive bed ballistocardiography in cardiovascular monitoring, Clin. Physiol. 16 (1996) 23-30.

[30] D.D. He, E.S. Winokur, C.G. Sodini, A continuous, wearable, and wireless heart monitor using head ballistocardiogram (BCG) and head electrocardiogram (ECG), in: Proceedings of the 33rd Annu. Int. Conf. of the IEEE EMBS, Boston, Massachusetts, USA, 2011, pp. 4729-4732.

Please cite this article in press as: C. Alvarado-Serrano, et al., An algorithm for beat-to-beat heart rate detection from the BCG based on the continuous spline wavelet transform, Biomed. Signal Process. Control (2015), http://dx.doi.org/10.1016/j.bspc.2016.02.002 